bioRxiv preprint doi: https://doi.org/10.1101/2021.11.02.466989; this version posted November 4, 2021. The copyright holder for this preprint (which was not certified by peer review) is the author/funder, who has granted bioRxiv a license to display the preprint in perpetuity. It is made available under aCC-BY-ND 4.0 International license.

\title{
Validating the wearable MUSE headset for EEG spectral analysis and Frontal Alpha Asymmetry*
}

*Research supported by a grant from the Institute of Noetic Sciences and the REGION OCCITANIE, France.

Cédric Cannard

The Brain and Cognition Research Center (CerCo), CNRS, Paul Sabatier

University, Toulouse, France

Insitute of Noetic Sciences (IONS), Petaluma, USA

ccannard@protonmail.com orcid: 0000-0002-6125-1175
Helané Wahbeh

Insitute of Noetic Sciences (IONS), Petaluma, USA

hwahbeh@noetic.org orcid: 0000-0003-3650-4633

\author{
The Brain and Cognition Research \\ Center (CerCo), CNRS, Paul Sabatier \\ University, Toulouse, France
}

Swartz Center of Computational Neuroscience (SCCN), INC, UCSD, La Jolla, USA

Insitute of Noetic Sciences (IONS), Petaluma, USA

arnodelorme@gmail.com orcid: 0000-0002-0799-3557

\begin{abstract}
EEG power spectral density (PSD), the individual alpha frequency (IAF) and the frontal alpha asymmetry (FAA) are all EEG spectral measures that have been widely used to evaluate cognitive and attentional processes in experimental and clinical settings, and that can be used for real-world applications (e.g., remote EEG monitoring, brain-computer interfaces, neurofeedback, neuromodulation, etc.). Potential applications remain limited by the high cost, low mobility, and long preparation times associated with high-density EEG recording systems. Low-density wearable systems address these issues and can increase access to larger and diversified samples. The present study tested whether a low-cost, 4-channel wearable EEG system (the MUSE) could be used to quickly measure continuous EEG data, yielding similar frequency components compared to research a grade EEG system (the 64-channel BIOSEMI Active Two). We compare the spectral measures from MUSE EEG data referenced to mastoids to those from BIOSEMI EEG data with two different references for validation. A minimal amount of data was deliberately collected to test the feasibility for real-world applications (EEG setup and data collection being completed in under $5 \mathrm{~min}$ ). We show that the MUSE can be used to examine power spectral density (PSD) in all frequency bands, the individual alpha frequency (IAF; i.e., peak alpha frequency and alpha center of gravity), and frontal alpha asymmetry. Furthermore, we observed satisfying internal consistency reliability in alpha power and asymmetry measures recorded with the MUSE. Estimating asymmetry on PAF and CoG frequencies did not yield significant advantages relative to the traditional method (whole alpha band). These findings should advance human neurophysiological monitoring using wearable neurotechnologies in large participant samples and increase the feasibility of their implementation in real-world settings.
\end{abstract}

Keywords - wearable EEG, power spectral density, frequency domain, signal validation, frontal alpha asymmetry, individual alpha frequency (IAF).

\section{INTRODUCTION}

The MUSE (InterAxon Inc.) is a low-cost, off-the-shelf, wearable EEG headset that has two frontal and two temporoparietal (TP) dry active EEG channels. It has been validated for evoked-response potential (ERP) research (i.e., time-domain; [1]) and used in many recent studies [2]-[12]. However, to our knowledge, it has not yet been validated for frequency domain analysis (power spectra on continuous EEG data), with one study showing mixed results [13]. In addition to assessing the validation of MUSE spectral measures, it is relevant to test if the MUSE could be used to estimate clinically- and research- relevant spectral measures, such as the frontal alpha asymmetry (FAA) and the individual alpha frequency (IAF).

Frontal alpha asymmetry (FAA; or frontal EEG asymmetry) refers to the relative difference in log alpha power $(8-12 \mathrm{~Hz})$ between the right and the left frontal regions. This spectral measure has been widely used to evaluate participants' cognitive, emotional, and attentional processes, both as an event-related state response and as a trait during rest [14][19]. Because of the inhibitory role of alpha oscillations [20][23], relatively greater left than right alpha power is associated with relatively greater right than left cortical activity. In addition, greater activation of the left-frontal cortex relative to the right is related to approach motivation and emotions with positive valence (e.g., happiness, positive urgency), whereas greater activation of the right-frontal cortex relative to the left is associated with the brain processes related to avoidance motivation and negative emotional valence (e.g., depression, anxiety, withdrawal). FAA is suspected to reflect neural processes of the executive control systems and has been source-localized to the frontoparietal network [19].

The individual alpha frequency (IAF) refers to the frequency within the alpha band with dominant spectral power [24]. It is associated with cognitive performance [25], considered a trait-like characteristic of human EEG [26], has high heritability and test-retest reliability [27], [28], and better accounts for interindividual differences in alpha activity [24], [29]. It has been traditionally examined using the peak alpha frequency (PAF) approach, which takes the frequency with the highest alpha power within the alpha band [30]-[32]. However, it has been highlighted that this approach does not perform well in a large portion of the population (up to 44\%) that displays absent, ambiguous, or "split" alpha peaks [24], 
bioRxiv preprint doi: https://doi.org/10.1101/2021.11.02.466989; this version posted November 4, 2021. The copyright holder for this preprint (which was not certified by peer review) is the author/funder, who has granted bioRxiv a license to display the preprint in perpetuity. It is made available under aCC-BY-ND 4.0 International license.

[33]. The alpha center of gravity ( $\mathrm{CoG})$ is considered a more robust approach to calculate the IAF by considering the whole alpha power distribution [24].

The IAF may be used to estimate FAA. Since alpha power distribution can fall outside the traditional predefined range (8-13 Hz) for some individuals [32], asymmetry scores based on the IAF (instead of the traditional band) might better address interindividual differences and might therefore provide more accurate asymmetry indexes method for research and clinical applications [34], [35].

IAFs and FAA seem like promising candidate measures for wearable EEG systems, as they require simple calculations in the frequency domain and a few EEG channels covering the frontal regions of each hemisphere. While these measures have not been validated using these systems against researchgrade EEG, wearable EEG systems have been used extensively over the past few years to measure frontal asymmetry, suggesting this measure is well-suited for these technologies [2], [36]-[45]. Wearable systems, when reliable, can offer advantages for researchers through easeful EEG data collection for over large samples, increased access to populations that are hard to study with conventional systems (e.g., children, elderly, patients), reduced hardware and software costs, and facilitated EEG research in real-world environments by increasing subjects' mobility and streaming the data wirelessly [46].

However, there is still a lack of validation of the data collected by such devices and the interpretation of the results based on the literature based on conventional higher-density systems and different referencing methods (i.e., linkedmastoids or average reference). The reference method implemented for low-density wearable systems is of particular importance when considering measuring EEG asymmetry [47], [48]. Both IAF and FAA are promising EEG measures for neurofeedback applications [35], [49], which would benefit from mobile data collection.

The present study tested whether the 4-channel wearable MUSE EEG system can quickly measure continuous EEG data with a maximum of 5-minute set-up and data collection time, that would yield quantifiable frequency components comparable to research-grade systems and if it can extract clinically relevant measures such as IAF and FAA.

\section{METHODS}

\section{A. Participants}

Participants for this study were 40 English-speaking adults in the San Francisco Bay area. Exclusion criteria were: aged younger than 18 years old, unable to read, having an acute or chronic illness that interfered with the completion of the experiment, or being unable to sit on a chair for about 30 minutes. Participants had their EEG recorded with a 64channel EEG system at the laboratory for another study $(\sim 2 \mathrm{~h}$ session) and were asked if they wanted to volunteer a few more minutes of their time for an additional $~ 5$ minutes EEG recording using the wearable headset. They were compensated only for their participation in the initial study. They gave informed consent, and the study was approved by the IONS Institutional Review Board.

\section{B. EEG data collection procedures}

EEG data were collected with the active dry MUSE 1 (version 2016) at $256 \mathrm{~Hz}$ and a 64-channel gel-based BIOSEMI Active 2 system (BIOSEMI Inc.) at $512 \mathrm{~Hz}$. Simultaneous recording of both systems was not possible due to their configurations. The MUSE data were recorded first, and then the BIOSEMI data about 30 minutes later, which corresponded to the time necessary to set up the BIOSEMI equipment and optimize channel impedance). A comparison of the two systems' hardware specifications can be found in Table 1. For both systems, the participants' skin was cleaned with alcohol wipes at electrode sites before positioning the headband/head cap.

MUSE - A thin layer of water was applied to the dry electrodes with a sponge for both the frontal metallic sensor and the conductive silicone rubber mastoid sensors behind the ears to decrease the impedance and increase signal quality. The MindMonitor App [50] running on a Chromebook laptop was used to record the EEG signal and check electrode contact (a colored circle for each electrode was filled when the software deemed the connection acceptable). Visual examination of the raw EEG waveforms was also performed while participants were asked to generate eye blinks to provide an additional index of signal quality. The headset position was adjusted if the signal was judged too noisy by visual inspection of the data.

BIOSEMI - Highly conductive electrolytes SignaGel was injected into the electrode sites of the BIOSEMI head cap. BIOSEMI active electrode offsets were kept below offset 20 using the Actiview software.

TABLE I. HARDWARE SPECIFICATIONS OF EACH SYSTEM

\begin{tabular}{|c|c|c|}
\hline & Biosemi Active Two & InteraXon MUSE \\
\hline $\begin{array}{c}\text { Electrode } \\
\text { montage }\end{array}$ & $\begin{array}{c}64 \text { wet active electrodes (10-20 } \\
\text { system) }\end{array}$ & $\begin{array}{l}4 \text { dry active electrodes } \\
(\text { AF7, AF8, TP9, TP10) }\end{array}$ \\
\hline $\begin{array}{l}\text { Sampling } \\
\text { rate }\end{array}$ & $512 \mathrm{~Hz}$ & $256 \mathrm{~Hz}$ \\
\hline Resolution & 24 bits & 12 bits \\
\hline $\begin{array}{l}\text { Active } \\
\text { electrode } \\
\text { system }\end{array}$ & $\begin{array}{l}\text { Passive DRL and active CMS } \\
\text { located around } \mathrm{POz}\end{array}$ & $\begin{array}{l}\text { Passive DRL and active } \\
\text { CMS located at Fpz }\end{array}$ \\
\hline Head sizes & $\begin{array}{l}3 \text { different head cap sizes } \\
\text { covering } 54-62 \mathrm{~cm}\end{array}$ & $\begin{array}{l}\text { Adjustable headband, 52-60 } \\
\mathrm{cm} \text { range }\end{array}$ \\
\hline $\begin{array}{l}\text { Recording } \\
\text { apparatus }\end{array}$ & $\begin{array}{c}\text { Optic fiber and amplifier, } \\
\text { MacBook Pro, Actiview } \\
\text { Software }\end{array}$ & $\begin{array}{l}\text { Bluetooth on a low-cost } \\
\text { Chromebook, Mind } \\
\text { Monitor App }\end{array}$ \\
\hline Reference & Reference free ${ }^{a}$ & Fpz \\
\hline
\end{tabular}

Not choosing a reference led to a $40 \mathrm{~dB}$ loss of signal-to-noise (SNR) ratio

Not choosing a reference

MUSE and BIOSEMI - Recordings were performed at the same location within the recording room, minimizing the differences in terms of potential electrical artifacts from the environment. One minute of data was recorded with eyes open gazing at the computer screen in front of them, and one minute was recorded with eyes closed. Half the participants did eyes open before eyes closed, and the other half did the reversed order to avoid carry-over effects. Participants were instructed to sit still on a chair, limit their movements, and 
bioRxiv preprint doi: https://doi.org/10.1101/2021.11.02.466989; this version posted November 4, 2021. The copyright holder for this preprint (which was not certified by peer review) is the author/funder, who has granted bioRxiv a license to display the preprint in perpetuity. It is made available under aCC-BY-ND 4.0 International license.

focus their attention on their breath by counting each inhalation/exhalation cycle. In this manuscript, we only process eyes' closed data.

\section{EEG data preprocessing}

BIOSEMI data were imported into the EEGLAB processing software (v2021.1; [51]) using the BIOSIG plugin (v3.7.5). MUSE data were imported using the MUSEMonitor (v3.2) plugin of EEGLAB. BIOSEMI data were downsampled to $256 \mathrm{~Hz}$. Raw data were high-pass filtered with EEGLAB's linear non-causal Finite Impulse Response (FIR) filter of the FIRFILT (v2.4) plugin (filter order $=1129$; transition bandwidth $=0.75 \mathrm{~Hz}$; passband edge $=0.75 \mathrm{~Hz} ;-6 \mathrm{~dB}$ cutoff frequency $=0.375 \mathrm{~Hz}$ ). No low-pass filter was used.

Files were inspected visually for abnormal channels (bad connection, impedance, very high noise, flat sections from disconnections, etc.) and artifactual segments (eye and muscle artifacts, high-frequency bursts, etc.). Artifactual regions and channels were manually rejected. MUSE data files with at least 1 visually abnormal channel were removed. If the BIOSEMI or the MUSE file was shorter than $45 \mathrm{~s}$, the participant data was also excluded from further analysis. Using these criteria, three out of 40 data files were excluded.

The traditional method to compute frontal alpha asymmetry (FAA) is to calculate the difference in log-transformed alpha power between the frontal electrodes F7 and F8 on 64channel EEG data [47], [48]. While the linked-mastoids reference method has been used extensively in the EEG asymmetry literature, average-referencing was shown to be preferable to estimate FAA [47]. Thus, spectral measures were obtained on BIOSEMI data referenced to the average (called the "average-ref montage" in this study) and on BIOSEMI re-referenced to mastoids (called the "mastoid-ref montage"). With 4 electrodes, an average reference is not meaningful for the MUSE system since it requires a wholehead electrode coverage. The default reference channel for the MUSE is Fpz which is close to the frontal channels AF7 and AF8, and leads to low signal amplitude on these channels. Thus, the MUSE frontal channels were re-referenced to the TP9/TP10 mastoid electrodes (the two other channels available on the MUSE), termed in this study the "mastoidref montage" (AF7 and AF8 with linked mastoid reference)". This reference method has been widely used in the asymmetry literature (e.g., [47], [52]).

To assess if spectral measures obtained with the MUSE mastoid-ref montage are reliable and interpretable in terms of underlying neural activity, we tested whether they were comparable to those obtained with the BIOSEMI mastoid-ref montage and the BIOSEMI average-ref montage.

\section{Power spectral density (PSD)}

Power spectral density (PSD) was computed using the pwelch function in MATLAB 2021a (The MathWorks Inc., MA, United States) for each EEG channel on 4-second hamming windows, with $50 \%$ overlap and $200 \%$ padding (taking into account data discontinuity due to excluded artifactual regions). The mean was removed from PSD data, and they were converted to decibels $\left(10 * \log _{10}(\right.$ power $\left.)\right)$ [48]. Mean PSD was extracted for each frontal channel for each frequency band: delta $(1-3 \mathrm{~Hz})$, theta $(3-7 \mathrm{~Hz})$, alpha (8-13
$\mathrm{Hz})$, beta $(14-30 \mathrm{~Hz})$, and gamma $(>30 \mathrm{~Hz})$. Then, the average between the two channels was used for analyses.

\section{E. Individual alpha frequency $(I A F)$}

Both the peak alpha frequency (PAF) and the alpha center of gravity (CoG) were estimated using the open-source and automated restingIAF toolbox (v1.0.2; [24]). This method uses curve-fitting algorithms, zero-crossing, and SavitzkyGolay Filter (SGF) smoothing techniques (same parameters as above for PSD estimation, a minimum of 1 required channel to estimate PAF and $\mathrm{CoG}$, and the default values for the other parameters).

\section{F. Frontal alpha asymmetry}

Three methods were used to calculate alpha asymmetry:

- Traditional method: the difference between the frontal channels on alpha power (in $\mathrm{dB}$ ) averaged over the 8$13 \mathrm{~Hz}$ band (mean_alpha_right - mean_alpha_left).

- PAF-asymmetry. Same as above but on power at the peak alpha frequency (PAF).

- CoG-asymmetry. Same as above but on power at the alpha center of gravity (CoG).

\section{G. Internal consistency reliability}

Previous research showed that reliable asymmetry values can be obtained with as little as 80 seconds of data [53]. To confirm internal consistency reliability of the asymmetry measures with the different montage methods and with very short segments of data ( 45 seconds for the shortest file after data cleaning), mean alpha power and FAA (traditional method only) were also computed for each montage on eleven 4-s blocks of data (mean for each block). Internal consistency reliability of alpha PSD and FAA was evaluated using Cronbach's standardized alpha on the blocks of spectral data [54], [55]. Values $>.8$ indicate high internal consistency reliability and $<.3$ indicate low internal consistency reliability; [53].

\section{H. Statistics}

All spectral measures were compared using the skipped Pearson correlation from the open-source Robust Correlation MATLAB toolbox [56]. Skipped Pearson correlations detect and remove bivariate outliers using the minimum covariance determinant (MCD) estimator, and better control for the type I error by accounting for their deletion when testing for significance, and by using bootstrapped $95 \%$ confidence intervals $(C I$; [56]-[58]). If the $C I$ encompasses 0 , then the null hypothesis $(\mathrm{HO})$ of independence cannot be rejected. This approach is less sensitive to heteroscedasticity (i.e., change in the spread of the residuals over the range of measured values leading to biased results) and therefore more robust against the type I error [56], [57]. Rejections of $H O$ at the $95 \%$ confidence level (i.e., significant correlations) are reported next to the skipped Pearson correlation $r$ coefficient scores with * (i.e., $p<0.05$ ). Bivariate outliers correspond to the red observations in the plots. The red line correspond to the least square fit line, and the red shaded areas correspond to the $95 \% \mathrm{CI}$. 
bioRxiv preprint doi: https://doi.org/10.1101/2021.11.02.466989; this version posted November 4, 2021. The copyright holder for this preprint (which was not certified by peer review) is the author/funder, who has granted bioRxiv a license to display the preprint in perpetuity. It is made available under aCC-BY-ND 4.0 International license.

\section{RESULTS}

\section{A. Internal consistency reliability}

The following Cronbach's alpha scores were obtained for frontal alpha power (.98 - BIOSEMI average-ref montage; .95 - MUSE mastoid-ref montage) and frontal alpha asymmetry (.67 - BIOSEMI average-ref montage; .76 MUSE mastoid-ref montage).

\section{B. Power spectral density (PSD) for each frequency band}

The averaged PSD of each frequency band was first compared between the BIOSEMI mastoid-ref montage and the MUSE mastoid-ref montage. All frequency bands were significantly correlated between the two montages: delta (1$3 \mathrm{~Hz}, \mathrm{r}=.59^{*}$, CI $\left.[0.38,0.75]\right)$, theta $\left(3-7 \mathrm{~Hz}, \mathrm{r}=.73^{*}, \mathrm{CI}\right.$ $[0.55,0.85])$, alpha $\left(8-13 \mathrm{~Hz}, \mathrm{r}=.87^{*}\right.$, CI $\left.[0.77,0.93]\right)$, beta $\left(14-30 \mathrm{~Hz}, \mathrm{r}=.84^{*}\right.$, CI $\left.[0.70,0.91]\right)$, and gamma $(>30 \mathrm{~Hz}, \mathrm{r}$ $=0.48^{*}$, CI $\left.[0.19,0.69]\right)$. These results are plotted in Fig. 1 .

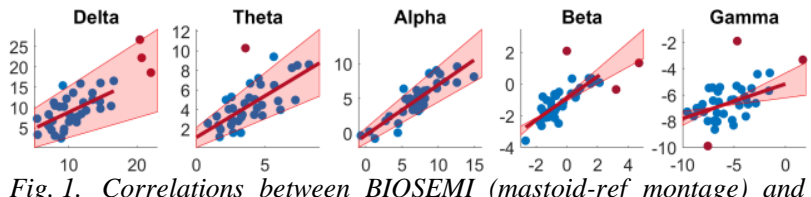

Fig. 1. Correlations between BIOSEMI (mastoid-ref montage) and MUSE (mastoid-ref montage) of mean power spectral density (PSD) for each frequency band: delta $(1-3 \mathrm{~Hz})$, theta $(3-7 \mathrm{~Hz})$, alpha $(8-13 \mathrm{~Hz})$, beta $(14-30 \mathrm{~Hz})$, and gamma (> $30 \mathrm{~Hz})$. All frequency bands were significantly correlated. Statistics are reported in the text of the Results section. Red dots are bivariate outliers accounted for by the skipped Pearson correlations. The red line is the least-squares fit line. Shaded areas are the 95\% confidence intervals. The power spectral density (PSD) unit is deciBels $\left(10 * \log 10\left(\mu \mathrm{V}^{2} / \mathrm{Hz}\right)\right)$.

Correlations between PSD estimates from MUSE mastoid-ref montage and those from BIOSEMI average-ref montage are reported in Fig. 2. Significant correlations were observed for the delta $\left(\mathrm{r}=.47^{*}\right.$, CI $\left.[0.19,0.69]\right)$, the theta $\left(\mathrm{r}=.63^{*}\right.$, CI $[0.43,0.78])$, the alpha $(\mathrm{r}=.80 *$, CI $[0.65,0.90]$, and the beta $(\mathrm{r}=.74 *$, CI $[0.58,0.86])$ bands. However, the correlation was not significant for the gamma band $(r=.17$, CI $[-0.13$, $0.50])$.

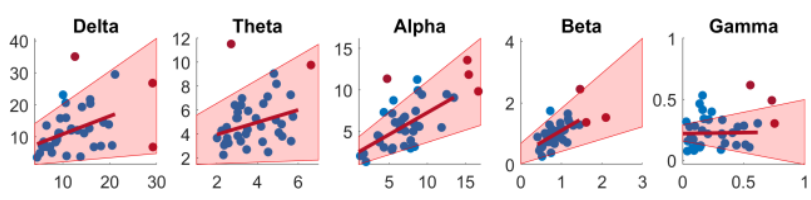

Fig. 2. Correlations between BIOSEMI (average-ref montage) and MUSE (mastoid-ref montage) of mean power spectral density (PSD) for each frequency band: delta $(1-3 \mathrm{~Hz})$, theta $(3-7 \mathrm{~Hz})$, alpha $(8-13 \mathrm{~Hz})$, beta $(14-30 \mathrm{~Hz})$, and gamma (> $30 \mathrm{~Hz})$. All frequency bands except gamma were significantly correlated. Statistics are reported in the text of the Results section. Red dots are bivariate outliers accounted for by the skipped Pearson correlations. The red line is the least-squares fit line. Shaded areas are the $95 \%$ confidence intervals. The power spectral density (PSD) unit is deciBels $\left(10 * \log 10\left(\mu \mathrm{V}^{2} / \mathrm{Hz}\right)\right)$.

\section{Individual alpha frequency $(I A F)$}

IAFs estimated on BIOSEMI mastoid-ref montage were significantly correlated with those obtained on MUSE mastoid-ref montage (Fig. 3, left), for both PAF ( $\mathrm{r}=.91^{*}$, CI $[0.79,0.97])$ and CoG $\left(r=.78^{*}\right.$, CI $\left.[0.64,0.88]\right)$. However, PAF could not be estimated on BIOSEMI for 7 files, and for
MUSE on 13 files. CoG could not be estimated on BIOSEMI data for 5 files and on MUSE data for 4 files.

Correlations between IAF for the BIOSEMI average-ref montage and the MUSE mastoid-ref montage (Fig. 3, right) were also significant for both estimation methods: PAF $(\mathrm{r}=$ $\left..95^{*}, \mathrm{CI}[0.86,0.98]\right)$ and $\mathrm{CoG}\left(\mathrm{r}=.84^{*}\right.$, CI $\left.[0.69,0.93]\right)$. However, the automated algorithms could not detect the PAF for 18 files (11 on BIOSEMI data and 13 on MUSE data) and the CoG for 6 files ( 5 for BIOSEMI and 4 for MUSE).
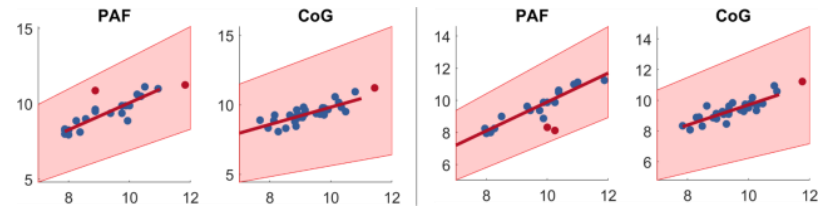

Fig. 3. Left: Correlations between BIOSEMI (mastoid-ref montage) and MUSE (mastoid-ref montage) of individual alpha frequency (IAF). Right:between BIOSEMI (average-ref montage) and MUSE (mastoidref montage). All estimates using both the peak alpha frequency (PAF) and the alpha center of gravity ( $\mathrm{CoG})$ were significantly correlated between the two systems and montages. Statistics are reported in the text of the Results section. Red dots are bivariate outliers accounted for by the skipped Pearson correlations. The red line is the least-squares fit line. Shaded areas are the $95 \%$ confidence intervals. The power spectral density (PSD) unit is deciBels $\left(10^{*} \log 10\left(\mu \mathrm{V}^{2} / \mathrm{Hz}\right)\right)$.

\section{Frontal alpha asymmetry (FAA)}

The three methods to compute FAA were significantly correlated between BIOSEMI and MUSE with the same mastoid-ref montage: traditional asymmetry $\left(\mathrm{r}=.67^{*}, \mathrm{CI}\right.$ $[0.40,0.93])$, PAF-asymmetry $\left(r=.35^{*}, \mathrm{CI}[0.7,0.62]\right.$, CoGasymmetry $(\mathrm{r}=0.42 *$, CI $[0.05,0.69])$. These results are plotted in Fig. 4.
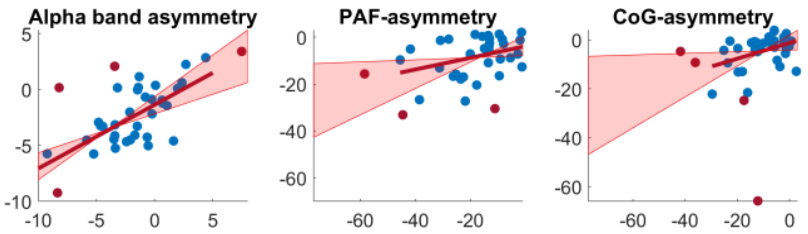

Fig. 4. Comparison of frontal alpha asymmetry measures from BIOSEMI mastoid-ref montage and MUSE mastoid-ref montage. The three forms of frontal alpha asymmetry were significantly correlated between the two systems. Statistics are reported in the text. Red dots are bivariate outliers accounted for by the skipped Pearson correlations. The red line is the least-squares fit line. Shaded areas are the $95 \%$ confidence intervals. The power spectral density (PSD) unit is deciBels $\left(10 * \log 10\left(\mu \mathrm{V}^{2} / \mathrm{Hz}\right)\right)$.

Finally, FAA measures were compared between the BIOSEMI average-ref montage and the MUSE mastoidref montage and are plotted in Fig. 5. FAA calculated on the average power over the whole alpha band (i.e., traditional method) was significantly correlated $\left(r=.37^{*}\right.$, CI $[0.06$, $0.60]$ ). However, asymmetry scores calculated on power at the PAF $(\mathrm{r}=.12$, CI $[-0.24,0.44])$ and at the $\mathrm{CoG}(\mathrm{r}=.26$, CI $[-0.02,0.55])$ were not significantly correlated. 

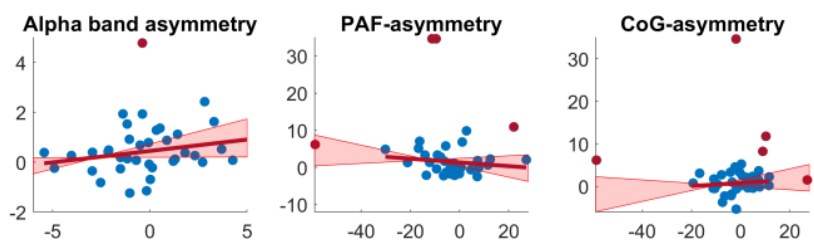

Fig. 5. Comparison of frontal alpha asymmetry measures from BIOSEMI average-ref montage and MUSE mastoid-ref montage. Alpha asymmetry calculated using the traditional method (on average power over the whole alpha band) was significantly correlated between the two systems. However, asymmetry scores calculated on the PAF and CoG power were not significantly correlated. Statistics are reported in the text. Red dots are bivariate outliers accounted for by the skipped Pearson correlations. The red line is the least-squares fit line. Shaded areas are the $95 \%$ confidence intervals. The power spectral density (PSD) unit is deciBels $\left(10 * \log 10\left(\mu \mathrm{V}^{2} / \mathrm{Hz}\right)\right)$.

\section{DISCUSSION}

\section{A. Results and interpretations}

When comparing MUSE mastoid-ref montage with BIOSEMI mastoid-ref montage, all spectral measures significantly correlated, indicating that this low-cost wearable EEG system can accurately capture these frequency components and that interpretations are in line with the literature using mastoid-ref montages can be made. However, correlation coefficients and CIs indicate that the traditional method to calculate frontal alpha asymmetry should be preferred relative to the PAF- and CoG- asymmetry methods.

When comparing MUSE mastoid-ref montage with BIOSEMI average-ref montage, PSD (in all frequencies beow $30 \mathrm{~Hz}$ ), IAF, and FAA (traditional method) were significantly correlated, indicating that the MUSE can be used to examine these measures and interpret the findings in line with the literature using the average-ref montages (i.e., F7 and F8 sites referenced to average). PAF- and CoGasymmetry measures were not significantly correlated, indicating they should only be interpreted in the mastoid-ref montage context.

These latter findings may suggest that:

1. The automated toolbox used for IAF-estimation does not perform well on low-density sparse montages and is better suited for higher density montages (since it can use neighboring channels to improve detection performance; [24]). Channels referenced to average may have contained alpha spectral components from other channels that were not captured by the mastoid-ref montage. IAF measures (PAF and $\mathrm{CoG}$ ) could not be estimated for some files, which could have reduced statistical power compared to the traditional measures. However, the superior performance of the CoG method compared to the PAF method was apparent since it was able to find IAF in many more participants.

2. The traditional asymmetry method is more robust and grounded in theory (independently of the montage). Previous research suggested that EEG asymmetry is influenced by different neural processes between the lower and the upper frequencies of the alpha band [32]. Thus, while IAFs better account for interindividual differences and are associated with some cognitive processes (e.g., memory), they might reflect different underlying neural processes than those underlying alpha asymmetry (e.g., executive control, attention, emotion regulation). Thus IAF-asymmetries might not be wellsuited for asymmetry calculation.

\section{B. Limitations}

The first limitation of this study is the 30 minutes difference between the two recordings. Mental states may likely have changed between the two recordings. However, correlations were still significant when comparing the MUSE and the BIOSEMI with the same mastoid-ref montage, suggesting trait spectral components were still captured. Ideally, both types of data should have been recorded simultaneously using markers to synchronize the data at the millisecond resolution. While this was not possible for this study, future studies should aim to record both systems simultaneously.

Second, FAA during rest was previously estimated to vary $\sim 60 \%$ from trait influence and $40 \%$ from state influences [59], the former being the target measure in this study. While internal consistency reliability of asymmetry measures was relatively high, more variation and lower values were observed compared to the internal consistency reliability of the alpha power data (as in previous publications; [53]). Increasing the data length (e.g., 3 minutes of artifact-free data) might increase the trait influence by reducing the fluctuations due to state influences, and in turn, increase internal consistency reliability. We purposely used short segments to determine if they could be easily and reliably used in experimental and clinical conditions, but we did not compare different data lengths and their impact on these trait EEG measures. Future studies should compare asymmetry measures from a clinical system and a low-cost wearable system (as in this study) with longer data lengths to address this potential limitation.

The absence of correlation in the higher frequencies (PSD > $30 \mathrm{~Hz}$ ) when comparing MUSE with BIOSEMI average-ref montage but not mastoid-ref montage may suggest that these frequencies may reflect field potentials from other brain processes when referenced to average than those captured with the mastoid-ref montage. Thus, these frequencies should only be interpreted in the mastoid-ref montage context when uing this system.

\section{Recommendations for research and clinical MUSE recordings}

Recommendations for using the MUSE in future clinical and experimental research are as follows:

- Eye's closed recordings of at least 1 minute (ideally 5 if time allows), corresponding to a total preparation and recording time of about 3 minutes (about 8 for 5 -minute recordings).

- Cleaning the participants' skin with alcohol wipes and wetting the dry electrodes to reduce impedance.

- Manual exclusion of channels and bad data portions after data collection (or validation of an automated method on this system's signal).

- Re-referencing the data to linked mastoid electrodes (i.e., TP9/TP10).

- Using measures found to be reliable with this system: PSD $<30 \mathrm{~Hz}$, traditional FAA, and the IAF (in particular the $\mathrm{CoG})$. 
bioRxiv preprint doi: https://doi.org/10.1101/2021.11.02.466989; this version posted November 4, 2021. The copyright holder for this preprint (which was not certified by peer review) is the author/funder, who has granted bioRxiv a license to display the preprint in perpetuity. It is made available under aCC-BY-ND 4.0 International license.

- Robust statistical methods should be used to better account for outliers and higher noise in the spectral estimates that might occur more frequently using this montage and system (e.g., skipped correlations, iteratively reweighted least squares regressions or weighted least squares regressions)

\section{CONCLUSION}

Our study validates the use of the low-cost MUSE headset for accurately and reliably measuring PSD, IAFs, and FAA (calculated on the whole band). This system can help advance human neurophysiological monitoring techniques on large datasets using wearable neurotechnologies and increase the feasibility of their implementation into real-world applications.

\section{REFERENCES}

[1] O. E. Krigolson, C. C. Williams, A. Norton, C. D. Hassall, and F. L. Colino, "Choosing MUSE: Validation of a Low-Cost, Portable EEG System for ERP Research," Front. Neurosci., vol. 11, 2017, doi: 10.3389/fnins.2017.00109.

[2] A. Hashemi et al., "Characterizing Population EEG Dynamics throughout Adulthood," eNeuro, vol. 3, no. 6, p. ENEURO.027516.2016, Nov. 2016, doi: 10.1523/ENEURO.0275-16.2016.

[3] J. Amores, R. Richer, N. Zhao, P. Maes, and B. M. Eskofier, "Promoting relaxation using virtual reality, olfactory interfaces and wearable EEG," in 2018 IEEE 15th International Conference on Wearable and Implantable Body Sensor Networks (BSN), Mar. 2018, pp. 98-101. doi: 10.1109/BSN.2018.8329668.

[4] A. Arsalan, M. Majid, A. R. Butt, and S. M. Anwar, "Classification of Perceived Mental Stress Using A Commercially Available EEG Headband," IEEE J. Biomed. Health Inform., vol. 23, no. 6, pp. 2257-2264, Nov. 2019, doi: 10.1109/JBHI.2019.2926407.

[5] A. Asif, M. Majid, and S. M. Anwar, "Human stress classification using EEG signals in response to music tracks," Comput. Biol. Med., vol. 107, pp. 182-196, Apr. 2019, doi: 10.1016/j.compbiomed.2019.02.015.

[6] K. A. Cochrane, L. Loke, C. de Bérigny, and A. Campbell, "Sounds in the moment: designing an interactive EEG nature soundscape for novice mindfulness meditators," in Proceedings of the 30th Australian Conference on Computer-Human Interaction, New York, NY, USA, Dec. 2018, pp. 298-302. doi: 10.1145/3292147.3292215.

[7] K. Herman, L. Ciechanowski, and A. Przegalińska, "Emotional Well-Being in Urban Wilderness: Assessing States of Calmness and Alertness in Informal Green Spaces (IGSs) with Muse-Portable EEG Headband," Sustainability, vol. 13, no. 4, Art. no. 4, Jan. 2021, doi: 10.3390/su13042212.

[8] H. Hunkin, D. L. King, and I. T. Zajac, "Evaluating the feasibility of a consumer-grade wearable EEG headband to aid assessment of state and trait mindfulness," J. Clin. Psychol., vol. n/a, no. n/a, 2021, doi: 10.1002/jclp. 23189 .

[9] O. E. Krigolson et al., "Using Muse: Rapid Mobile Assessment of Brain Performance," Front. Neurosci., vol. 15, p. 634147, Jan. 2021, doi: 10.3389/fnins.2021.634147.

[10] O. E. Krigolson, C. C. Williams, and F. L. Colino, "Using Portable EEG to Assess Human Visual Attention," in Augmented Cognition. Neurocognition and Machine Learning, Springer, Cham, 2017, pp. 56-65. doi: 10.1007/978-3-319-58628-1_5.

[11] M. Papakostas, K. Tsiakas, T. Giannakopoulos, and F. Makedon, "Towards predicting task performance from EEG signals," in 2017 IEEE International Conference on Big Data (Big Data), Dec. 2017, pp. 4423-4425. doi: 10.1109/BigData.2017.8258478.
[12] X. Qu, Q. Mei, P. Liu, and T. Hickey, "Using EEG to Distinguish Between Writing and Typing for the Same Cognitive Task," in Brain Function Assessment in Learning, Cham, 2020, pp. 66-74. doi: 10.1007/978-3-030-60735-7_7.

[13] E. Ratti, S. Waninger, C. Berka, G. Ruffini, and A. Verma, "Comparison of Medical and Consumer Wireless EEG Systems for Use in Clinical Trials," Front. Hum. Neurosci., vol. 11, p. 398, 2017, doi: 10.3389/fnhum.2017.00398.

[14] J. J. Allen and J. P. Kline, "Frontal EEG asymmetry, emotion, and psychopathology: the first, and the next 25 years," Biol Psychol, vol. 67, no. 1-2, pp. 1-5, Oct. 2004.

[15] J. A. Coan and J. J. Allen, "Frontal EEG asymmetry as a moderator and mediator of emotion," Biol Psychol, vol. 67, no. 1-2, pp. 7-49, Oct. 2004.

[16] E. Harmon-Jones, P. A. Gable, and C. K. Peterson, "The role of asymmetric frontal cortical activity in emotion-related phenomena: a review and update," Biol Psychol 19733618, vol. 84, no. 3, pp. 45162, Jul. 2010, doi: S0301-0511(09)00182-3 [pii] 10.1016/j.biopsycho.2009.08.010.

[17] R. J. Davidson, "What does the prefrontal cortex 'do' in affect: perspectives on frontal EEG asymmetry research," Biol. Psychol., vol. 67, no. 1, pp. 219-234, Oct. 2004, doi: 10.1016/j.biopsycho.2004.03.008.

[18] R. Thibodeau, R. S. Jorgensen, and S. Kim, "Depression, anxiety, and resting frontal EEG asymmetry: a meta-analytic review," $J$ Abnorm Psychol 17100529, vol. 115, no. 4, pp. 715-29, Nov. 2006.

[19] G. M. Grimshaw and D. Carmel, “An asymmetric inhibition model of hemispheric differences in emotional processing," Front. Psychol., vol. 5, 2014, doi: 10.3389/fpsyg.2014.00489.

[20] H. Laufs et al., "EEG-correlated fMRI of human alpha activity," Neurolmage, vol. 19, no. 4, pp. 1463-1476, Aug. 2003, doi: 10.1016/S1053-8119(03)00286-6.

[21] H. Laufs et al., "Where the BOLD signal goes when alpha EEG leaves," Neuroimage, vol. 31, no. 4, pp. 1408-18, Jul. 2006.

[22] K. E. Mathewson, A. Lleras, D. M. Beck, M. Fabiani, T. Ro, and G. Gratton, "Pulsed Out of Awareness: EEG Alpha Oscillations Represent a Pulsed-Inhibition of Ongoing Cortical Processing," Front. Psychol., vol. 2, May 2011, doi: 10.3389/fpsyg.2011.00099.

[23] T. R. Oakes et al., "Functional coupling of simultaneous electrical and metabolic activity in the human brain," Hum. Brain Mapp., vol. 21, no. 4, pp. 257-270, Apr. 2004, doi: 10.1002/hbm.20004.

[24] A. W. Corcoran, P. M. Alday, M. Schlesewsky, and I. BornkesselSchlesewsky, "Towards a reliable, automated method of individual alpha frequency (IAF) quantification," Bioinformatics, preprint, Aug. 2017. doi: 10.1101/176792.

[25] E. Angelakis, J. F. Lubar, and S. Stathopoulou, "Electroencephalographic peak alpha frequency correlates of cognitive traits," Neurosci. Lett. 15500967, vol. 371, no. 1, pp. 603 , Nov. 2004, doi: S0304-3940(04)01052-3 [pii] 10.1016/j.neulet.2004.08.041

[26] T. H. Grandy, M. Werkle-Bergner, C. Chicherio, F. Schmiedek, M. Lövdén, and U. Lindenberger, "Peak individual alpha frequency qualifies as a stable neurophysiological trait marker in healthy younger and older adults: Alpha stability," Psychophysiology, vol. 50, no. 6, pp. 570-582, Jun. 2013, doi: 10.1111/psyp.12043.

[27] M. Näpflin, M. Wildi, and J. Sarnthein, “Test-retest reliability of resting EEG spectra validates a statistical signature of persons," Clin. Neurophysiol., vol. 118, no. 11, pp. 2519-2524, Nov. 2007, doi: 10.1016/j.clinph.2007.07.022.

[28] C. M. Smit, M. J. Wright, N. K. Hansell, G. M. Geffen, and N. G. Martin, "Genetic variation of individual alpha frequency (IAF) and alpha power in a large adolescent twin sample," Int. J. Psychophysiol., vol. 61, no. 2, pp. 235-243, Aug. 2006, doi: 10.1016/j.ijpsycho.2005.10.004. 
bioRxiv preprint doi: https://doi.org/10.1101/2021.11.02.466989; this version posted November 4, 2021. The copyright holder for this preprint (which was not certified by peer review) is the author/funder, who has granted bioRxiv a license to display the preprint in perpetuity. It is made available under aCC-BY-ND 4.0 International license.

[29] S. Haegens, H. Cousijn, G. Wallis, P. J. Harrison, and A. C. Nobre, "Inter- and intra-individual variability in alpha peak frequency," NeuroImage, vol. 92, pp. 46-55, May 2014, doi: 10.1016/j.neuroimage.2014.01.049.

[30] W. Klimesch, "EEG alpha and theta oscillations reflect cognitive and memory performance: a review and analysis," Brain Res. Brain Res. Rev. 10209231, vol. 29, no. 2-3, pp. 169-95, Apr. 1999.

[31] W. Klimesch, H. Schimke, G. Ladurner, and G. Pfurtscheller, "Alpha frequency and memory performance," J. Psychophysiol., vol. 4, no. 4, pp. 381-390, 1990.

[32] W. Klimesch, "EEG-alpha rhythms and memory processes," Int. J. Psychophysiol. 9203012, vol. 26, no. 1-3, pp. 319-40, Jun. 1997.

[33] A. K. I. Chiang et al., "Automated characterization of multiple alpha peaks in multi-site electroencephalograms," J. Neurosci. Methods, vol. 168, no. 2, pp. 396-411, Mar. 2008, doi: 10.1016/j.jneumeth.2007.11.001.

[34] C. W. E. M. Quaedflieg, T. Meyer, F. T. Y. Smulders, and T. Smeets, "The functional role of individual-alpha based frontal asymmetry in stress responding," Biol. Psychol., vol. 104, pp. 75-81, Jan. 2015, doi: 10.1016/j.biopsycho.2014.11.014.

[35] C. W. Quaedflieg, F. T. Smulders, T. Meyer, F. Peeters, H. Merckelbach, and T. Smeets, "The validity of individual frontal alpha asymmetry EEG neurofeedback," Soc Cogn Affect Neurosci, vol. 11, no. 1, pp. 33-43, Jan. 2016, doi: 10.1093/scan/nsv090.

[36] P. Arpaia, N. Moccaldi, R. Prevete, I. Sannino, and A. Tedesco, "A Wearable EEG Instrument for Real-Time Frontal Asymmetry Monitoring in Worker Stress Analysis," IEEE Trans. Instrum. Meas., vol. 69, no. $10, \quad$ p. $8335-8343$, Oct. 2020, doi: 10.1109/TIM.2020.2988744.

[37] Z. Cao, C.-T. Lin, W. Ding, M.-H. Chen, C.-T. Li, and T.-P. Su, "Identifying Ketamine Responses in Treatment-Resistant Depression Using a Wearable Forehead EEG," IEEE Trans. Biomed. Eng., vol. 66, no. 6, pp. 1668-1679, Jun. 2019, doi: 10.1109/TBME.2018.2877651

[38] S. Hwang, H. Jebelli, B. Choi, M. Choi, and S. Lee, "Measuring Workers' Emotional State during Construction Tasks Using Wearable EEG," J. Constr. Eng. Manag., vol. 144, no. 7, p. 04018050, Jul. 2018, doi: 10.1061/(ASCE)CO.1943-7862.0001506.

[39] H. Jebelli, S. Hwang, and S. Lee, "Feasibility of Field Measurement of Construction Workers' Valence Using a Wearable EEG Device," pp. 99-106, Jun. 2017, doi: 10.1061/9780784480830.013.

[40] H. Jebelli, M. M. Khalili, S. Hwang, and S. Lee, "A Supervised Learning-Based Construction Workers' Stress Recognition Using a Wearable Electroencephalography (EEG) Device," in Construction Research Congress 2018, New Orleans, Louisiana, Mar. 2018, pp. 40-50. doi: 10.1061/9780784481288.005.

[41] S. Park, C.-H. Han, and C.-H. Im, "Design of Wearable EEG Devices Specialized for Passive Brain-Computer Interface Applications," Sensors, vol. 20, no. 16, Art. no. 16, Jan. 2020, doi: $10.3390 / \mathrm{s} 20164572$.

[42] H. Peng, D. Majoe, and T. Kaegi-Trachsel, "Design and application of a novel wearable EEG system for e-healthcare," in Proceedings of 2011 international workshop on Ubiquitous affective awareness and intelligent interaction, New York, NY, USA, Sep. 2011, pp. 1-8. doi: 10.1145/2030092.2030094.

[43] S. M. U. Saeed, S. M. Anwar, H. Khalid, M. Majid, and U. Bagci, "EEG Based Classification of Long-Term Stress Using Psychological Labeling," Sensors, vol. 20, no. 7, Art. no. 7, Jan. 2020, doi: 10.3390/s20071886.

[44] S. M. Umar Saeed, S. M. Anwar, M. Majid, M. Awais, and M. Alnowami, "Selection of Neural Oscillatory Features for Human Stress Classification with Single Channel EEG Headset," BioMed
Res. Int., vol. 2018, p. e1049257, Dec. 2018, doi: $10.1155 / 2018 / 1049257$.

[45] S. Wu, X. Xu, L. Shu, and B. Hu, "Estimation of valence of emotion using two frontal EEG channels," in 2017 IEEE International Conference on Bioinformatics and Biomedicine (BIBM), Nov. 2017, pp. 1127-1130. doi: 10.1109/BIBM.2017.8217815.

[46] C. Cannard, T. Brandmeyer, H. Wahbeh, and A. Delorme, "Selfhealth monitoring and wearable neurotechnologies," Handb. Clin. Neurol., vol. 168, pp. 207-232, 2020, doi: 10.1016/B978-0-44463934-9.00016-0.

[47] E. E. Smith, S. J. Reznik, J. L. Stewart, and J. J. B. Allen, “Assessing and Conceptualizing Frontal EEG Asymmetry: An Updated Primer on Recording, Processing, Analyzing, and Interpreting Frontal Alpha Asymmetry," Int. J. Psychophysiol. Off. J. Int. Organ. Psychophysiol., vol. 111, pp. 98-114, Jan. 2017, doi: 10.1016/j.ijpsycho.2016.11.005.

[48] Allen, Coan, and M. Nazarian, "Issues and assumptions on the road from raw signals to metrics of frontal EEG asymmetry in emotion," Biol. Psychol., vol. 67, no. 1-2, pp. 183-218, Oct. 2004, doi: 10.1016/j.biopsycho.2004.03.007.

[49] E. Angelakis, S. Stathopoulou, J. L. Frymiare, D. L. Green, J. F. Lubar, and J. Kounios, "EEG neurofeedback: a brief overview and an example of peak alpha frequency training for cognitive enhancement in the elderly," Clin Neuropsychol 17366280, vol. 21, no. 1, pp. 110-29, Jan. 2007.

[50] J. Clutterbuck, "Mind Monitor," Mind Monitor, 2015. https://mindmonitor.com (accessed Oct. 27, 2021)

[51] A. Delorme and S. Makeig, "EEGLAB: an open source toolbox for analysis of single-trial EEG dynamics including independent component analysis," J. Neurosci. Methods, vol. 134, no. 1, pp. 921, 2004.

[52] J. L. Stewart, A. W. Bismark, D. N. Towers, J. A. Coan, and J. J. B. Allen, "Resting frontal EEG asymmetry as an endophenotype for depression risk: sex-specific patterns of frontal brain asymmetry," $J$. Abnorm. Psychol., vol. 119, no. 3, pp. 502-512, Aug. 2010, doi: 10.1037/a0019196.

[53] D. N. Towers and J. J. B. Allen, "A Better Estimate of the Internal Consistency Reliability of Frontal EEG Asymmetry Scores," Psychophysiology, vol. 46, no. 1, pp. 132-142, Jan. 2009, doi: 10.1111/j.1469-8986.2008.00759.x.

[54] L. J. Cronbach, "Coefficient alpha and the internal structure of tests," Psychometrika, vol. 16, no. 3, pp. 297-334, Sep. 1951, doi: 10.1007/BF02310555

[55] A. Schlegel, "Cronbach's Alpha," 2010. https://www.mathworks.com/matlabcentral/fileexchange/38320cronbach-s-alpha), MATLAB Central File Exchange. Retrieved October 27, 2021. (accessed Oct. 27, 2021).

[56] C. Pernet, R. Wilcox, and G. Rousselet, "Robust Correlation Analyses: False Positive and Power Validation Using a New Open Source Matlab Toolbox," Front. Psychol., vol. 3, p. 606, 2013, doi: 10.3389/fpsyg.2012.00606.

[57] R. R. Wilcox, G. A. Rousselet, and C. R. Pernet, "Improved methods for making inferences about multiple skipped correlations," J. Stat. Comput. Simul., vol. 88, no. 16, pp. 3116-3131, Nov. 2018, doi: 10.1080/00949655.2018.1501051.

[58] R. R. Wilcox, Introduction to robust estimation and hypothesis testing, 3rd ed. Amsterdam; Boston: Academic Press, 2012.

[59] D. Hagemann, E. Naumann, J. F. Thayer, and D. Bartussek, "Does resting electroencephalograph asymmetry reflect a trait? an application of latent state-trait theory," J Soc Psychol 11999928, vol. 82, no. 4, pp. 619-41, Apr. 2002. 